International Journal of Wireless \& Mobile Networks (IJWMN) Vol. 3, No. 5, October 2011

\title{
ENERGY CONSERVATION IN MANET USING VARIABLE RANGE LOCATION AIDED ROUTING PROTOCOL
}

\author{
Nivedita N. Joshi ${ }^{1}$ and Radhika D. Joshi ${ }^{2}$ \\ ${ }^{1}$ Department of Electronics and Tele-communication Engineering, College of \\ Engineering, Pune, India \\ niv.joshi@gmail.com \\ ${ }^{2}$ Department of Electronics and Tele-communication Engineering, College of \\ Engineering, Pune, India \\ rdj.extcacoep.ac.in
}

\begin{abstract}
A Mobile Ad-Hoc Network (MANET) is a temporary, infrastructure-less and distributed network having mobile nodes. MANET has limited resources like bandwidth and energy. Due to limited battery power nodes die out early and affect the network lifetime. To make network energy efficient, we have modified position based Location Aided Routing (LAR1) for energy conservation in MANET. The proposed protocol is known as Variable Range Energy aware Location Aided Routing (ELAR1-VAR). The proposed scheme controls the transmission power of a node according to the distance between the nodes. It also includes energy information on route request packet and selects the energy efficient path to route data packets. The comparative analysis of proposed scheme and LAR1 is done by using the QualNet simulator. ELAR1-VAR protocol improves the network lifetime by reducing energy consumption by $20 \%$ for dense and mobile network while maintaining the packet delivery ratio above $90 \%$.
\end{abstract}

\section{KEYWORDS}

MANET, LAR1, Packet Delivery Ratio, Energy Consumption

\section{INTRODUCTION}

Wireless communication is one of the fastest growing fields in Telecommunication Industry. These systems, such as cellular, cordless phones, wireless local area networks (WLAN) have become an essential tool for people in today's life. Using these systems and the equipments like PDA, laptops, cell phones user can access all the required information whenever and wherever needed. All these systems need some fixed infrastructure. It takes time and potentially high cost to set up the necessary infrastructure. There are situations where user required networking connections are not available in a given geographic area, and providing the needed connectivity and network services in these situations becomes a real challenge. So, in this situation mobile communication network without a pre-exist network infrastructure is the best solution. The Ad Hoc Networks are wireless networks characterized by the absence of fixed infrastructures. The main aim of mobile ad- hoc network (MANET) [1] is to support robust and efficient operation in mobile wireless networks.

MANET consists of mobile nodes which form a spontaneous network without a need of fixed infrastructure. It is an autonomous system in which mobile hosts connected by wireless links are free to move randomly and often act as routers at the same time. Hence, it forms multi-hop network. The ad-hoc networks are finding more importance likely due to the features that they

DOI : 10.5121/ijwmn.2011.3520 
can be easily deployed as well as reconfigured. This allows the use of this kind of network in special circumstances, such as disastrous events, the reduction or elimination of the wiring costs and the exchange of information among users independently from the environment. The applications for MANETs are ranging from large-scale, mobile, highly dynamic networks, to small, static networks that are constrained by power sources [2]. It can be used in military communication, commercial sectors like disaster management, emergency operations, wireless sensor networks, etc.

Deployment of ad-hoc network leads to many challenges such as limited battery power, limited bandwidth, multi hop routing, dynamic topology, security [1]. But, the major issue in MANET is energy consumption since nodes are usually mobile and battery-operated. Power failure of a mobile node affects its functionality thus the overall network lifetime. To prolong life time of the network, ad-hoc routing protocol should consider energy consumption. Efficient minimum energy routing schemes can greatly reduce energy consumption and extends the lifetime of the networks. Different routing protocols are designed for MANET such as AODV, DSR, OLSR, ZRP, and LAR, which meets some of challenges explained above. But, they do not consider the energy-efficient routing. Here, we are proposing a scheme which uses energy efficient routing to route the data. The modification is done in position based LAR1 protocol.

Rest of the paper is organized as: a Section II different routing approach for MANET is discussed. Section III gives the brief overview of LAR1 protocol. The energy efficient routing schemes are surveyed in Section IV. Section V discusses a proposed idea. Section VI gives the detail description of simulation model and performance metrics used for experimentation. Comparative analysis of proposed and original LAR1 protocols using QualNet simulator is given in section VII. Finally, Section VIII concludes the paper with future work in Section IX.

\section{DifFERENT ROUTING APPROACHES FOR MANET}

Many routing protocols have been developed for MANET with features like distributed operation, creation of loop free paths, security, and QoS support. MANET routing protocol is a standard that controls how nodes select the short and optimized route to route packets between communicating devices in a MANET. Routing protocols in MANET are broadly classified into proactive (Table-driven) and reactive (On-demand) routing protocols. These are based on routing strategy in MANET. Depending upon the network structure, the routing protocols are categorized as flat routing; hierarchical routing and geographic position assisted routing [3].

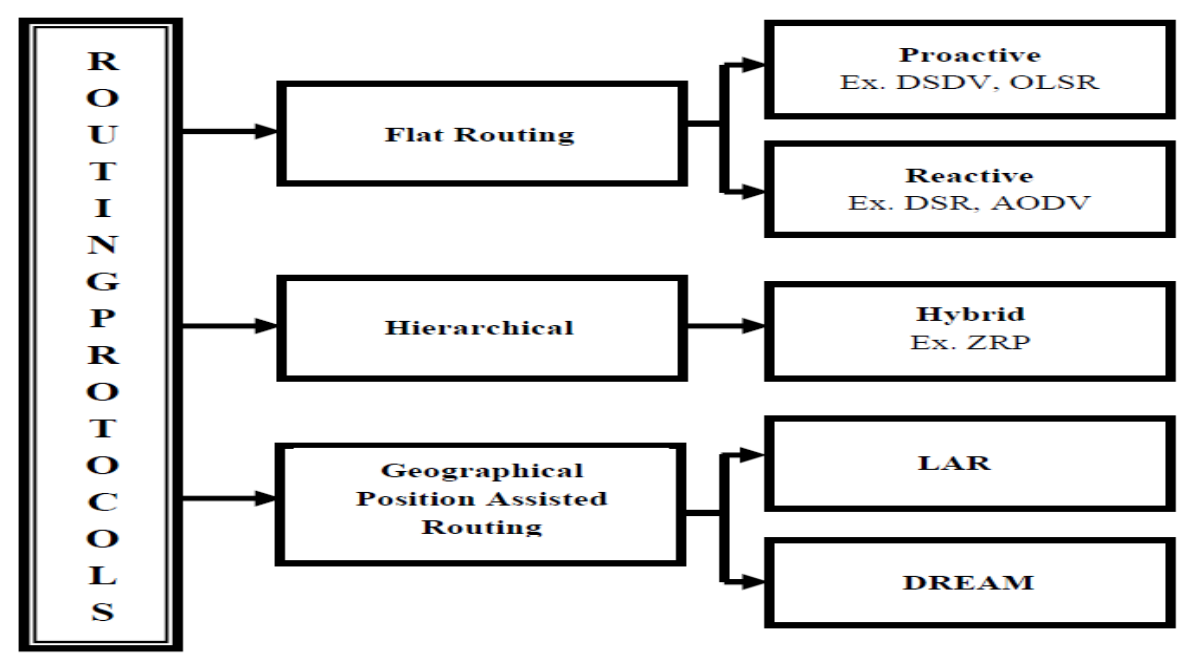

Figure 1. Classification of Routing Protocols 
International Journal of Wireless \& Mobile Networks (IJWMN) Vol. 3, No. 5, October 2011

\subsection{Proactive routing protocols}

Proactive routing protocols maintain information in a table on each node about the routing to the other node in the network. Although the topology of the network does not change, this information must be updated periodically. Many proactive routing protocols have been proposed, for e.g. Destination Sequence Distance Vector (DSDV), Optimized Linked State Routing (OLSR) and so on.

\subsection{Reactive protocols}

These protocols don't maintain routing information or routing activity at the network nodes if there is no communication. If a node wants to send a packet to another node then this protocol searches for the route in an on-demand manner and establishes the connection in order to transmit and receive the packet. The route discovery usually occurs by flooding the route request packets throughout the network. Several reactive protocols have been proposed such as Dynamic Source Routing protocol (DSR), Ad hoc On-demand Distance Vector (AODV), and so on.

\subsection{Hybrid routing protocols}

For larger networks, it is complex to design routing protocols. All nodes in the network are separated into groups, called cluster. All clusters form a hierarchical infrastructure. For such networks separate routing protocols have been designed which are known as hybrid routing protocols. These are the combination of proactive and reactive routing protocols. They are used in large networks in order to take advantages of proactive scheme for maintaining the routes in a cluster and reactive scheme for retaining the routes between the clusters. Several hybrid routing protocols have been proposed such as Zone Routing Protocol (ZRP), Zone-based Hierarchical Link State (ZHLS).

\subsection{Geographic position based Routing protocols}

The above mentioned types of MANET routing protocols do not consider the geographical location of a destination node. Position-based routing protocols do not establish or maintain route, a packet is forwarded one hop closer to its final destination by comparing the location of destination with the location of the node currently holding the packet. These routing protocols make minimum use of the topology information, hence, they exhibit better scalability compared to topology-based routing protocols. Such protocols use 'localized' algorithms like greedy forwarding, in which a node forwards a packet to a next hop that is geographically closest to the destination among its one-hop neighbors. Several Position-based protocols have been proposed such as Location aided Routing (LAR) [4], Distance Routing Effect Algorithm for Mobility (DREAM), Most Forward within Distance R (MFR).

\section{INTRODUCTION TO LAR1}

Position-based routing protocols exhibit better scalability, performance and robustness against frequent topological changes. These routing protocols use the geographical location of nodes to make routing in networks. This will improve efficiency and performance of the network. The main aim of Position-based LAR1 described in [4] is to reduce the control overheads by the use of location information.

\subsection{Location Information}

LAR1 protocol requires the information about geographical location of the nodes in network. This location information can be determined by using Global Positioning System (GPS) [5]. By using location information, LAR1protocol limits the search for a new route to a smaller request 
zone of the ad hoc network. This results in a significant reduction in the number of routing messages.

\subsection{Estimation of Expected and Request Zones}

LAR1 has two types of zone, Expected zone and Request zone, to restrict the flooding of route request packets. A source node uses the location service to find out the location of the destination and according to that information it will set the expected zone. Request zone is also determined by the source node and it is zone where a route request should be forwarded from source.

\subsubsection{Expected Zone}

Expected zone is set up by the source node $\mathrm{S}$ when it has data intended for destination node D. By using location service node $S$ estimates the geographical location of node $D$ at time $t_{0}$. Suppose node $\mathrm{D}$ was at location $\mathrm{O}$ at time $\mathrm{t}_{0}$, and that the current time $\mathrm{is}_{1}$. From this information node $S$ is capable of determining the 'expected zone' of node $\mathrm{D}$ from the viewpoint of node $S$ by time $t_{1}$. It is the region that node $S$ expects to contain node $D$ at time $t_{1}$. For instance, if node $\mathrm{D}$ is travelling with average speed $v$, then node $\mathrm{S}$ assumes that node $\mathrm{D}$ is in the expected zone of circular region of radius $v\left(t_{1}-t_{0}\right)$, centered at location $O$. The expected zone is only an estimate made by node $S$ to determine a region that may contain $D$ at time $t_{1}$. Since, if actual speed of node $\mathrm{D}$ is greater than the average, then the destination $\mathrm{D}$ may actually be outside the expected zone at time $t_{1}$. Figure 1 shows the expected zone created by the source node $\mathrm{S}$.

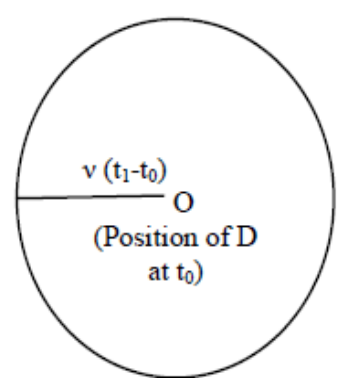

Figure 2. Expected Zone

\subsubsection{Request Zone}

The 'request zone' is different from the expected zone. It is the zone where a route request should be forwarded from source. An intermediate node will forward a route request packet only, if it belongs to the request zone. The request zone should contain the expected zone to reach destination node $\mathrm{D}$. The source node $\mathrm{S}$ defines this zone for flooding the route request packets. An intermediate node will forward the request packet, only if it is located within the request zone.

\subsection{Protocol Functioning}

In LAR1 scheme the request zone is of a rectangular shape. Assumption is that the source node $\mathrm{S}$ knows the destination node D's average speed $v$ and location $\left(\mathrm{X}_{\mathrm{d}}, \mathrm{Y}_{\mathrm{d}}\right)$ at time $\mathrm{t}_{0}$. The request zone is considered to be the smallest rectangle that includes the current source location, and the expected zone. The sides of the rectangle are parallel to $\mathrm{X}$ and $\mathrm{Y}$ axes. The source node determines the request zone and initiates the route request packet containing the four corners coordinates of request zone. Each intermediate node, receiving the request packet, checks whether it belongs to the rectangle; if it does not, it will discard the packet such as node Q does 
in the following Figure 2. In the same Figure 2, node $\mathrm{P}$ will forward the packet because it belongs to the rectangle. In the reply packet, node $\mathrm{D}$ will attach its accurate location and the current time stamp, which will be stored in the source's cache for future use.

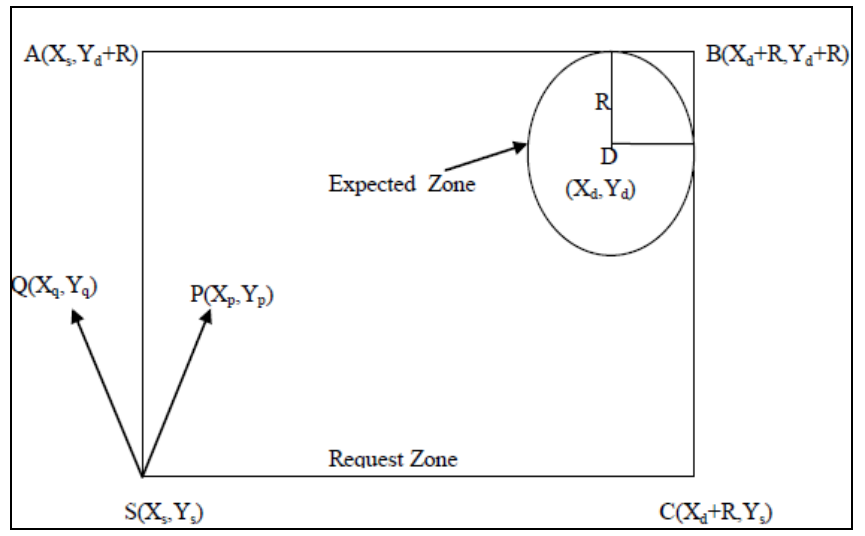

Figure 3. Working of LAR1

\section{BRIEF OVERVIEW OF ENERGy EFFICIENT ROUTING SCHEMES}

Several energy-efficient techniques are proposed to reduce energy consumption in MANET. These techniques use energy aware metrics to establish a path in a network. These metrics are residual energy, transmission power or link distance.

Niranjan Kumar Ray and Ashok Kumar Turuk have discussed different energy efficient techniques for wireless ad-hoc network [6]. One of the techniques is based on reduction of number of route request messages. In second Power control technique, next hop node is chosen depending on the power level of the node. Topology control technique is used to remove the energy-inefficient link from the network by examining the power level of the node. This technique helps network devices to take decision about their transmission range.

Morteza Maleki, Karthik Dantu, and Massoud Pedram in [7] have proposed a new power-aware source-initiated (on demand) routing protocol for mobile Ad-hoc networks that increase the network lifetime up to $30 \%$. A greedy policy was applied to fetched paths from the cache to make sure no path would be overused and also make sure that each selected path has minimum battery cost among all possible path between two nodes. Power-aware Source Routing (PSR) has taken care of both the node mobility and the node energy depletion that may cause a path to become invalid.

Energy-based Route Discovery Mechanism in Mobile Ad Hoc Networks [8] selects the route which has lowest energy cost in the network. The energy cost represents energy consumption of the network in order to prolong all connections between source and destination nodes. The energy cost is calculated using realistic energy consumption modeling which is used the channel quality to decide whether each packet is successfully received.

Location aided Energy-Efficient Routing protocol (LEER) protocol finds out the all possible paths from source to destination and selects minimum energy path to route the packets [9]. The selection of next hop node is based on whether it is situated near to destination than to source as well as transmit power of that node.

An energy aware routing scheme in location based ad-hoc network has proposed by Jangsu Lee, Seunghwan Yoo and Sungchun KimIn [10]. This method modifies the LAR protocol in which the virtual grid is applied to ad hoc network region and high energy node is selected as header for each grid which communicates information about nodes in that particular grid. The transmit 
power of nodes is adjusted according to the distance between them. The next hop node will be selected based on transmit power and its distance from the destination.

Nen-Chung Wang and Si-Ming Wang [11] have proposed a scheme which decides the baseline line between the source node and the destination node, for route discovery. The next hop is then selected based on baseline by broadcasting the request packets in request zone. The neighboring node with the shortest distance to the baseline is chosen as the next hop node. This method reduces control overheads by finding a better routing path than LAR scheme. They have proposed a partial reconstruction process for maintaining broken links of routing path.

Arthi Kothandaraman has proposed a protocol which based on transmission power control [12]. It varies the transmission range of a node to exclusively accommodate an independent node's neighbor set. This purely distributed as well as protocol independent scheme and preserves connectivity, and allows low power transmissions.

\section{Proposed Scheme}

LAR1 protocol uses location information of a node for setting the path from source to destination. We take this feature of LAR1 as a key factor in designing of variable range technique. The main aim is to design a technique of variable transmission power control to reduce overall energy consumption of the network. RREQ in LAR1 protocol consists of source location and destination location information. We have used this information to calculate the distance between the nodes. We also embed the energy factor of the node in RREQ packet for selection of energy efficient path.

\subsection{Energy Factor Calculation}

Yang Qin et. al. [13] have proposed an energy efficient routing metric called as Energy factor. They have used this metric for multipath concept where the most energy efficient as well as shortest path is selected to deliver the data packets. Similarly, in our method we use this metric to select the next hop node while discovering the path to destination. We define an energyefficient routing metric for selecting the node having sufficient energy to route the packets. The terms used in metric are as follows:

$\mathrm{EF}_{\mathrm{p}}$ : Energy Factor of node $\mathrm{p}$

$\mathrm{RE}_{\mathrm{p}}$ : Remaining Energy of node $\mathrm{p}$

$\mathrm{IE}_{\mathrm{p}}$ : Initial Energy of node $\mathrm{p}$

Energy factor is calculated by using the formula (1) for every node when it receives route request or data packet.

$$
E F p=R E p / I E p
$$

The EF of all the nodes along a valid path are multiplied together to obtain the EF of the path. We define it as minimum cost and it is given by-

$$
\text { Minimumcost }=\prod_{p \in N} E 5 p
$$

Where, N: Number of nodes between source S and Destination D.

Minimum cost metric selects the most energy-efficient path. The purpose of multiplication of $\mathrm{EF}$ values is to select the nodes having sufficient energy so that the minimum cost of the path from source to destination is high. 
International Journal of Wireless \& Mobile Networks (IJWMN) Vol. 3, No. 5, October 2011

\subsection{Flow Chart for ELAR1-VAR}

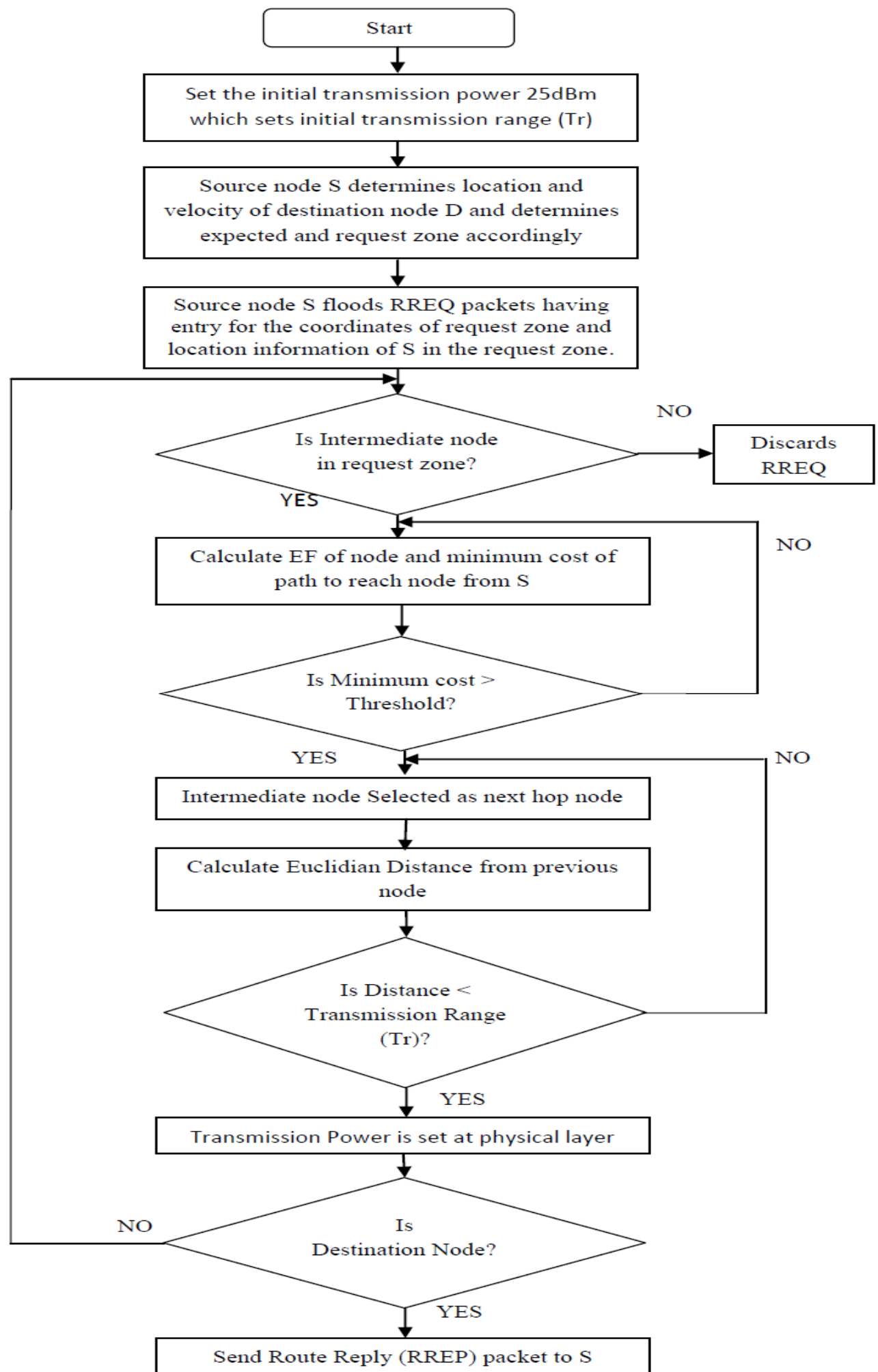

Figure 4. Flow Chart for ELAR-VAR 
International Journal of Wireless \& Mobile Networks (IJWMN) Vol. 3, No. 5, October 2011

\section{Simulation Model and Performance Metrics}

Simulator used for the comparative analysis is QualNet 5.0 [14]. It is suitable for designing new protocols, comparing different protocols and traffic evaluations. QualNet architecture is divided into three levels, Kernel, Model Libraries and Graphical User Interface (GUI).

1. Kernel- It provides the scalability and portability to run hundreds of nodes.

2. Model Libraries- Different types of model libraries enable to design networks using protocol models.

3. QualNet GUI- The user can use the QualNet GUI for creating and animating network scenarios, and analyzing their performance using the analyzer.

\subsection{Important features of QualNet -}

1. Speed- QualNet enables designers to run multiple scenarios by varying model, network, and traffic parameters in a short time.

2. Scalability- QualNet can model thousands of nodes by taking advantage of the latest hardware and parallel computing techniques.

3. Model Fidelity- QualNet uses highly detailed IEEE standards-based implementation of protocol models.

4. Portability- QualNet and its library of models run on a vast array of platforms, including Windows XP, Mac OS X, and Linux operating systems

5. Extensibility- QualNet can connect to other hardware and software applications, such as OTB, real networks to greatly enhancing the value of the network model.

\subsection{Simulation Model}

Simulation model is comprised of traffic model, mobility model, energy model, and battery model. The detailed description of all these are as follows:

\subsubsection{Traffic Model and Mobility Model}

Traffic is generated using constant bit rate (CBR). The packet size is limited to 512 bytes. The mobility model is used to describe the movement pattern of mobile users, and how their location, velocity and acceleration change over time. Random waypoint mobility model is used to give mobility to nodes in the network.

\subsubsection{Energy Model}

Energy model is used to analyze the overall energy consumption of the network. QualNet supports generic energy model [15]. We have used generic energy model for experimentation because it can estimate energy consumption in transmitter for the case of continuous and variable transmit power levels. Table 1, shows specifications of generic energy model. 
International Journal of Wireless \& Mobile Networks (IJWMN) Vol. 3, No. 5, October 2011

Table 1. Generic Energy Model

\begin{tabular}{|l|l|}
\hline Parameter & Values \\
\hline Power Amplifier Inefficiency factor & 6.5 \\
\hline Transmit Circuitry power Consumption & $100.0 \mathrm{~mW}$ \\
\hline Receive Circuitry power Consumption & $130.0 \mathrm{~mW}$ \\
\hline Idle Circuitry power Consumption & $120.0 \mathrm{~mW}$ \\
\hline Sleep Circuitry power Consumption & 0.0 \\
\hline Supply voltage (volt) & 6.5 \\
\hline
\end{tabular}

\subsubsection{Battery Model}

MANET consists of mobile nodes which are battery operated. QualNet has Linear Battery model which enables the analysis of the discharge behavior of the battery in mobile nodes.

\subsection{Performance Metrics}

Quality of a service is the performance level of a service offered by the network. The multimedia services demand for quick and better quality of data, it means the data rate and delay are the key factors for these services. For military applications, the security and reliable data delivery are of major concern. In sensor networks, the energy consumption and battery life would be the most important parameters. Different performance metrics are used for comparison are as given below-

\subsubsection{Packet Delivery Ratio}

It is the ratio of total number of data packets received successfully at destination to number of data packets generated at the source [16]. PDR values range from 0 to 1 . Higher PDR values decide the consistency of the protocol.

\subsubsection{End-to-End Delay}

The end to end delay is the average time interval between the generation of a packet at a source node and the successfully delivery of the packet at the destination node [16]. Low end to end delay gives better performance of the network.

\subsubsection{ECSDD (Energy Consumption per Successful Data Delivery)}

It is the ratio of total energy consumption to the number of data packets successfully delivered to the destination [17]. Lower the ECSDD values indicate that node uses less energy for data communication. This helps in extending the lifetime of node and thus overall network lifetime.

\subsubsection{Average energy consumption}

Energy consumed by all nodes in the network [17]. Energy consumption should be as low as possible so as to make fair utilization of limited battery power. 


\section{SimULATION RESULTS AND DISCUSSIONS}

The network area taken for experimentation is $1000 \mathrm{~m} \times 1000 \mathrm{~m}$. The comparison of proposed and original protocol is done for different number of nodes, speeds, packet rates and number of connections (Traffic load). Battery model is used at the node level with battery capacity $60 \mathrm{mAHr}$. We have set the transmission power for nodes equal to $25 \mathrm{dBm}$. The scenario model for examining the ELAR1-VAR in dense and moderately mobile network is given in Table 2.

Table 2. Scenario Model

\begin{tabular}{|l|l|l|l|l|}
\hline Parameters & $\begin{array}{l}\text { Variation of } \\
\text { Number of } \\
\text { Nodes }\end{array}$ & $\begin{array}{c}\text { Variation of } \\
\text { Node speed }\end{array}$ & $\begin{array}{l}\text { Variation of } \\
\text { Packet rate }\end{array}$ & $\begin{array}{l}\text { Variation of } \\
\text { Number of } \\
\text { connection }\end{array}$ \\
\hline Area $\left(\mathrm{m}^{2}\right)$ & $1000 \times 1000$ & $1000 \times 1000$ & $1000 \times 1000$ & $1000 \times 1000$ \\
\hline Nodes & $20,40,60,80,100$ & 50 & 50 & 50 \\
\hline Node & 10 & $1,5,10,15,20,25,30$ & 10 & 10 \\
\hline Simulation & 900 & 900 & 900 & 900 \\
\hline Pause Time(s) & 100 & 100 & 100 & 100 \\
\hline Traffic Type & $\mathrm{CBR}$ & $\mathrm{CBR}$ & $\mathrm{CBR}$ & $\mathrm{CBR}$ \\
\hline No. of & 25 & 25 & 25 & $25,30,35,40,45,50$ \\
\hline Packet & 4 & 4 & $2,4,6,8,10$ & 4 \\
\hline Initial Power & $60 \mathrm{mAHr}$ & $60 \mathrm{mAHr}$ & $60 \mathrm{mAHr}$ & $60 \mathrm{mAHr}$ \\
\hline
\end{tabular}

\subsection{Impact of Variation of Number of Nodes}

Simulation parameters for analysis of variation of number of nodes are given in Table 2. Both protocols are analyzed for variation of nodes from 20 to 100 . This will help in analyzing the behavior of protocols in dense and sparse network.

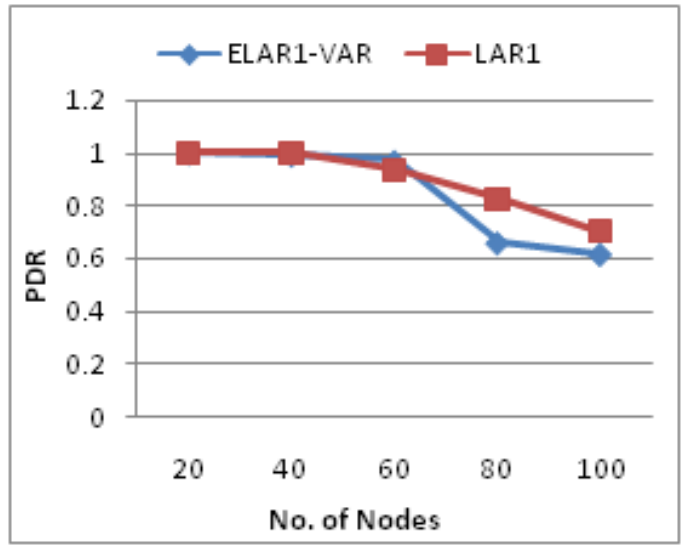

Figure 5. PDR vs. No. of Nodes

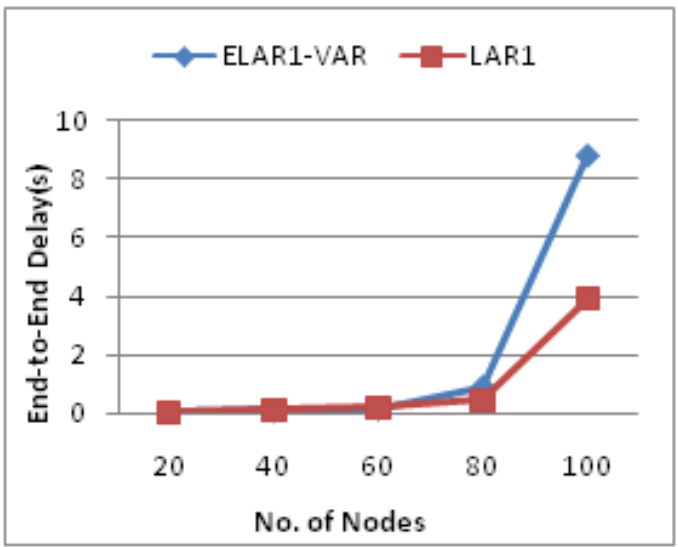

Figure 6. End-to-End Delay vs. No. of

Nodes

The variation of PDR and end to end delay with respect to number of nodes is shown in Figure 5 and Figure 6 respectively. It is observed that ELAR1-VAR has same packet delivery as LAR1 up to 60 nodes. PDR for highly dense network is slightly less. We can say that ELAR1-VAR is suitable option for low as well as dense network. End to end delay for modified protocol is equal to original one but for highly dense network it increases rapidly. This is because the 
modified method calculates the distances between the nodes for setting transmit power which increases the processing time of RREQ at intermediate node.

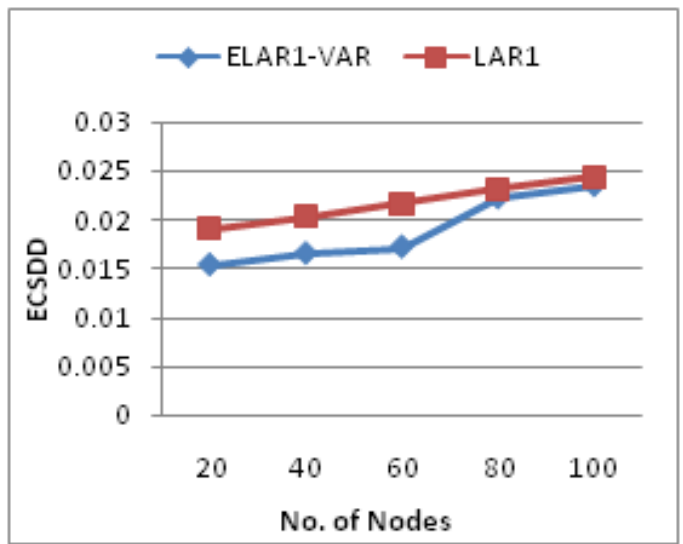

Figure 7. ECSDD vs. No. of Nodes

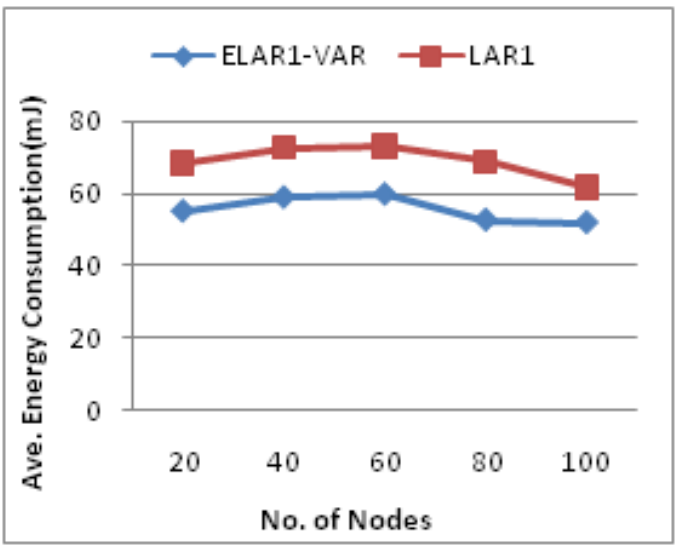

Figure 8. Ave. Energy Consumption vs. No. of Nodes

Figure 7 and Figure 8 shows ECSDD graph and energy consumed for different number of nodes respectively. The modified protocol consumes low energy for each successful data delivery. Hence, we can use ELAR1-VAR protocol for energy efficient applications. It is clear from figure 8 that energy consumption for ELAR1-VAR decreases approximately by $20 \%$.

\subsection{Impact of Variation of Node Speed}

We are varying the speed of mobile nodes from $1 \mathrm{~m} / \mathrm{s}$ to $30 \mathrm{~m} / \mathrm{s}$ to see the performance of ELAR1-VAR in mobile environment. The results for different performance metrics are as follows:

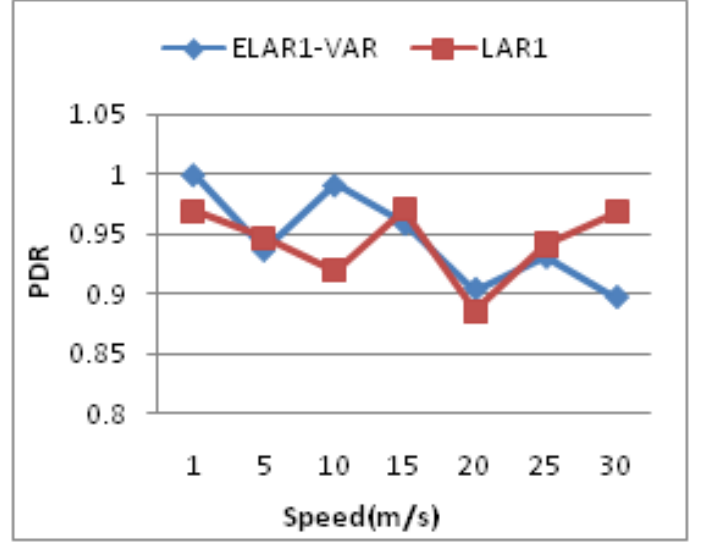

Figure 9. PDR vs. Speed

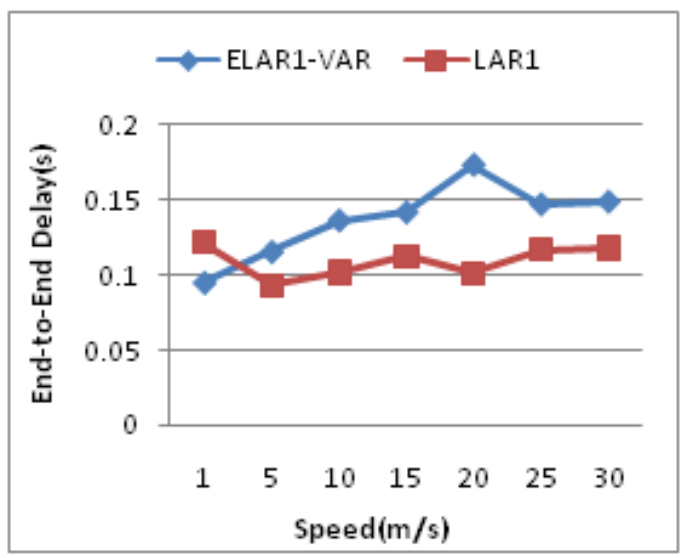

Figure 10. End-to-End Delay vs. Speed

The PDR and end to end delay results for different speeds are as shown in Figure 9 and Figure 10 respectively. If we increase the speed from 1 to 30 then PDR for ELAR1-VAR is approximately above $90 \%$ which indicates that it gives better results in low as well as high mobility network. There is an increase in delay for modified protocol. This is because location of node changes rapidly as node speed increases and node has to send new control packets which results in more delay. 


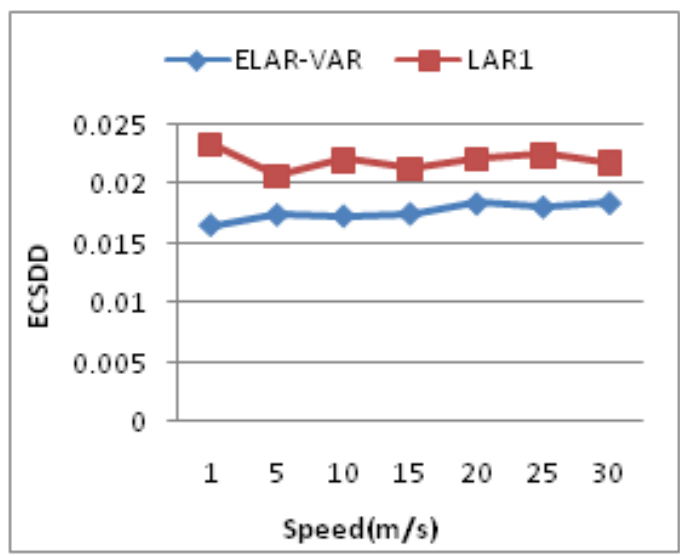

Figure 11. ECSDD vs. Speed

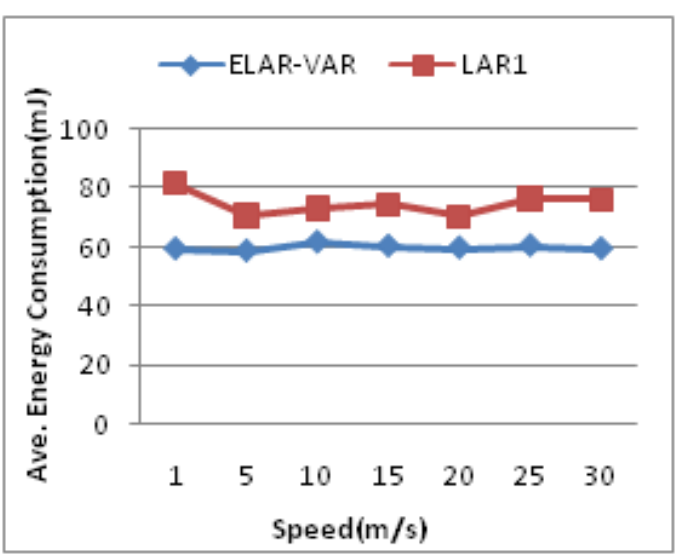

Figure 12. Average Energy Consumption vs. Speed

Impact of speed variation on ECSDD is shown in Figure 11. Energy consumption and ECSDD for ELAR1-VAR is less than LAR1. But, as speed increases the energy consumption in Figure 12 for both the protocol remains same. It is clear from Figure 11 that the energy consumption per data delivery decreases which improves the battery lifetime.

\subsection{Impact of Variation of Packet Rate}

Packet rate is varied from 2 to 10packets/second. Packet rate is varied to analyze the network capability of handling large number of data at a time.

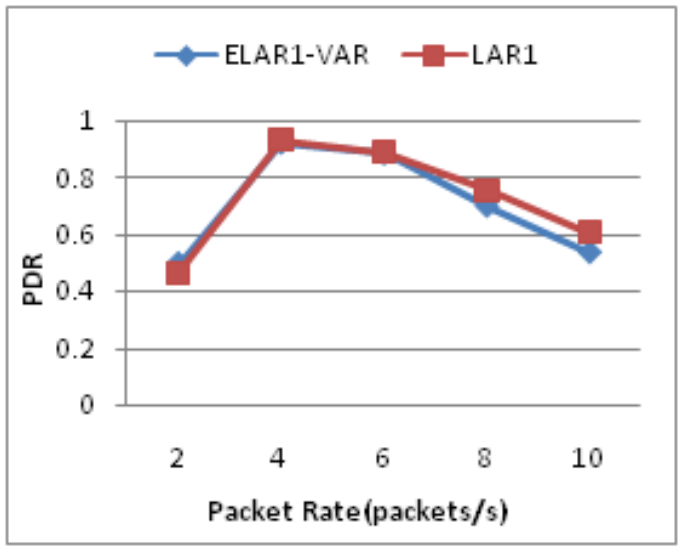

Figure 13. PDR vs. Packet Rate

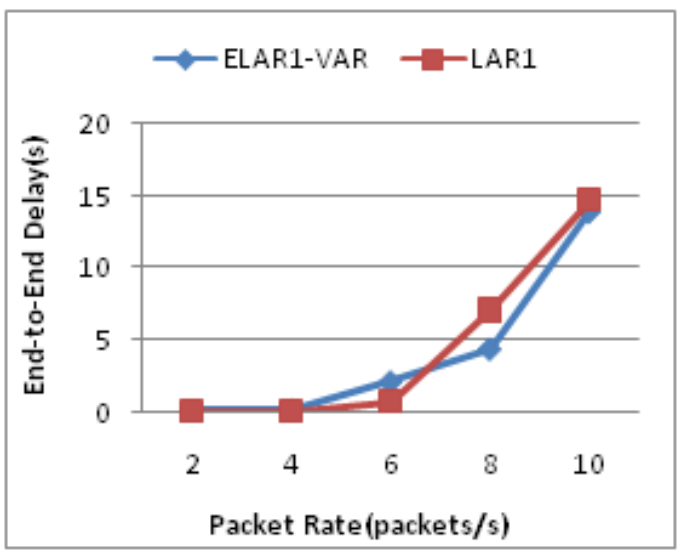

Figure 14. End-to-End Delay vs. Packet Rate

Figure 13 gives performance comparison of ELAR1-VAR with LAR1 in terms of PDR. There is increasing trend of PDR for both ELAR1-VAR and LAR1. As shown in Figure 14, ELAR1VAR has comparable delay value for lower packet rates. Delay increases with the packet rate for both protocols. Since, the more packets are to be sent at a time which overflows the buffer resulting in dropping of packets. Hence, packets are to be retransmitted which increases the end to end delay. 
International Journal of Wireless \& Mobile Networks (IJWMN) Vol. 3, No. 5, October 2011

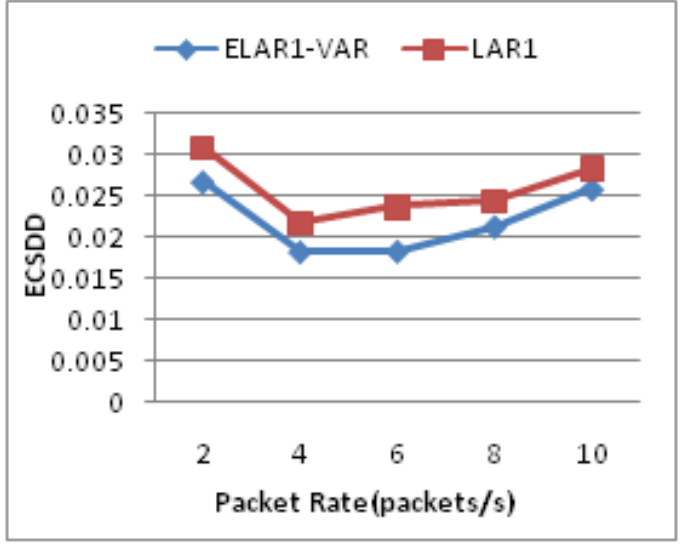

Figure 15. ECSDD vs. Packet Rate

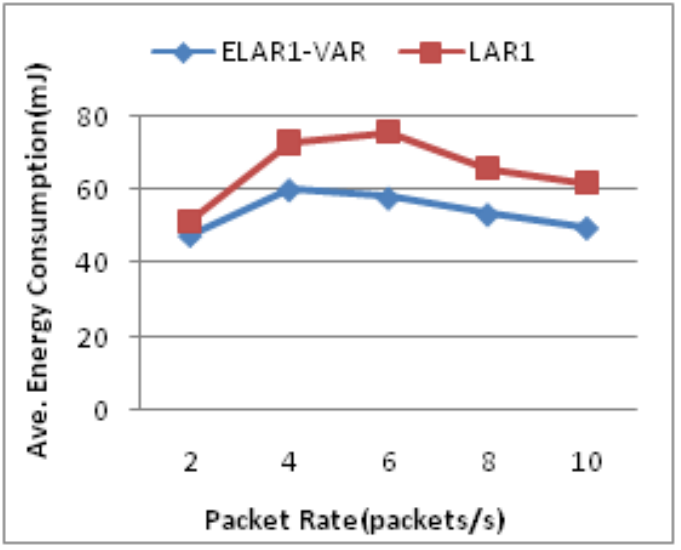

Figure 16. Average Energy Consumption vs. Packet Rate

As depicted in Figure 15, there is noteworthy change in ECSDD value for modified protocol. Also as shown in Figure 16, the overall energy consumption for network decreases with ELAR1-VAR.

\subsection{Impact of Variation of Number of Connections}

The number of connections i.e. source to destination data transfer connections are varied from 25 to 50 in steps of 5. This evaluation helps in understanding the behavior of network under variable traffic load.

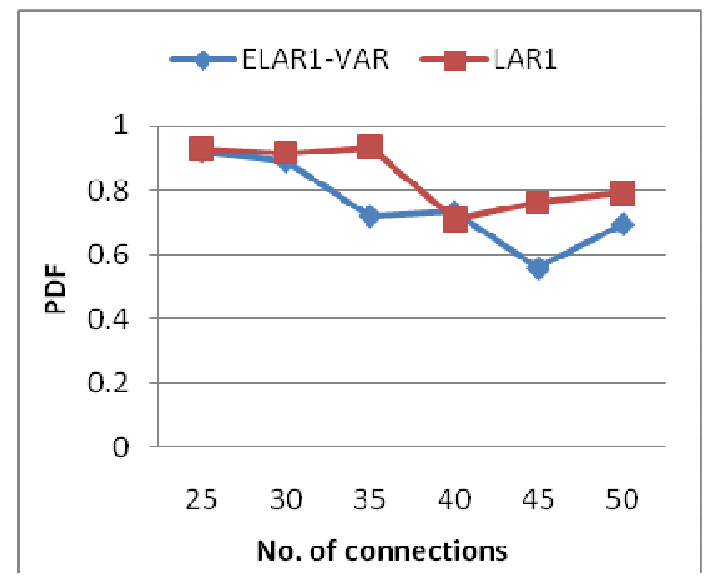

Figure 17. PDR vs. No. of connections

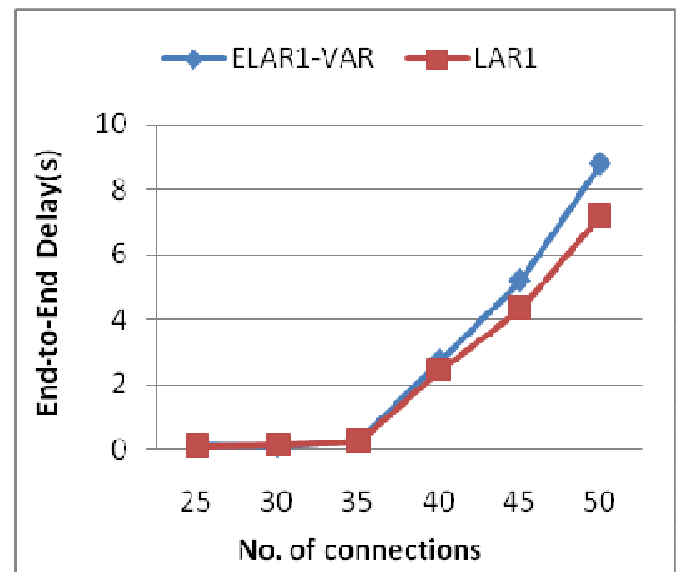

Figure 18. End-to-End Delay vs. No. of connections

Variation of PDR against number of connections is given in Figure 17. PDR decreases for both the protocols as traffic load increases. This happens due to the increase in traffic load creates the congestion in the network which will result into packets drop. End-to-end delay given in Figure 18 for LAR1 and ELAR-VAR1 is having same value up to 35 connections. For higher traffic loads; there is increasing trend for both the protocols. For the higher traffic loads, the queue at each node will be full and creating the congestion in the network. Thus, data delivery is delayed. 


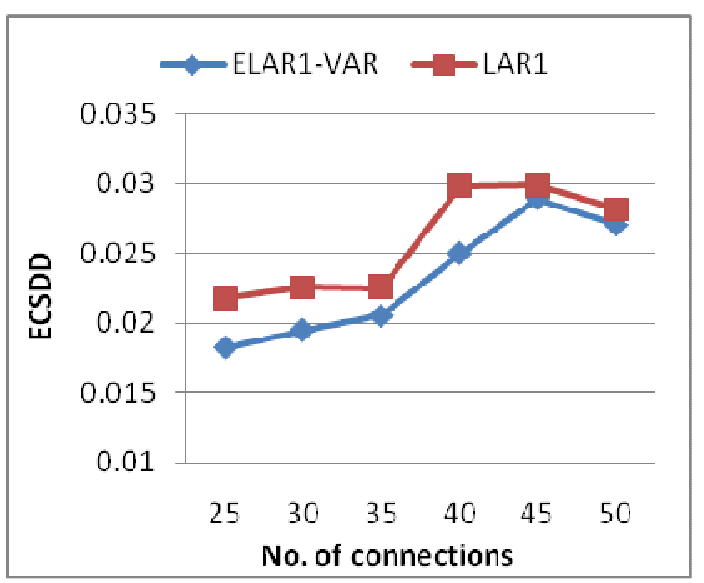

Figure 19. ECSDD vs. No. of connections

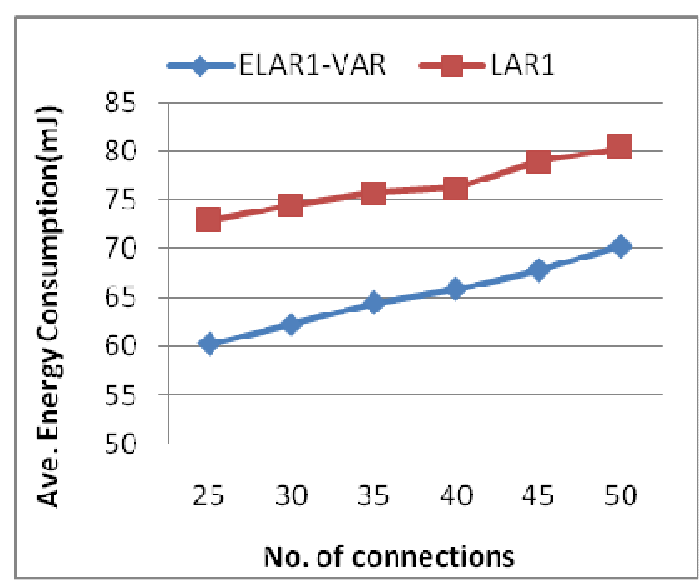

Figure 20. Average Energy Consumption vs. No. of connections

ECSDD and Average energy consumption graphs are given in Figure 19 and 20 respectively. Energy consumption increases linearly with the traffic load. For entire range of number of connections for ELAR1-VAR energy consumption is less as compared to LAR1. ECSDD values for the both protocols are remained almost constant up to $35 \mathrm{CBR}$ connections after that it increases with traffic load. ECSDD values are decreasing after 45 connections due increase in energy consumption and less packet delivery.

\section{CONCLusion}

We compared ELAR1-VAR and LAR1 protocols considering the performance metrics such as packet delivery ratio, end to end delay, average energy consumption, and ECSDD. Several simulations have performed under different network conditions to analyze the performance of modified protocol with LAR1. The analysis of results showed that there is very slight change in packet delivery but remarkable change in energy consumption. Packet delivery is low for highly dense network and for large traffic loads whereas for all speed variation it maintains $90 \%$ of PDR. Average end to end delay is less sensitive to node speed. Lower packet rate and traffic load have less impact on delay. It increases rapidly for higher packet rate and highly dense network.

The ELAR1-VAR consumes less energy for dense and moderately mobile network having packet rate of 4 packets/second. Network performance is better for traffic load about $50 \%$ of nodes i.e. for $25 \mathrm{CBR}$ connections. It is observed that overall energy consumption of the network is decreased by $20 \%$. In ELAR1-VAR, the energy consumption per successful data delivery is lowered and thus nodes in the network can communicate with each other for longer period. Thus, our aim of extending network lifetime is achieved. ELAR1-VAR due to its energy efficient feature can be used for the energy constrained applications.

\section{FUTURE Work}

The work can be extended to analyze the performance of ELAR1-VAR for different performance metrics such control overheads, jitter and throughput. 
International Journal of Wireless \& Mobile Networks (IJWMN) Vol. 3, No. 5, October 2011

In future, we will try to embed technique which will set threshold on the basis of nodes drain rate. This will help in selection of next hop node accurately.

\section{REFERENCES}

[1] Imrich Chlamtac, Marco Conti \& Jennifer J.-N. Liu, (2003) "Mobile ad hoc networking: imperatives and challenges", Ad-hoc Networks, Elsevier, pp13-64.

[2] Jun-Zhao Sun, (2001) "Mobile Ad Hoc Networking: An Essential Technology for Pervasive Computing”, International Conference on Info-tech and Info-net, Vol. 3, pp316-321.

[3] Krishna Gorantala, (2006) "Routing Protocols in Mobile Ad-hoc Networks", Master Thesis in Computer Science, pp1-36.

[4] Young-Bae Ko \& Nitin H. Vaidya, (1998) "Location-Aided Routing (LAR) in Mobile Ad Hoc Networks", MOBICOM 98, pp66-75.

[5] G. Dommety \& R. Jain, (1996) "Potential networking applications of global positioning systems (GPS)", Tech. Rep. TR-24, CS Dep., The Ohio State University.

[6] Niranjan Kumar Ray \& Ashok Kumar Turuk, (2010) "Energy Efficient Techniques for Wireless Ad Hoc Network", International Joint Conference on Information and Communication Technology, pp105-111.

[7] Morteza Maleki, Karthik Dantu \& Massoud Pedram, (2002) "Power-aware Source Routing Protocol for Mobile Ad Hoc Networks", Iternational Symposium on Low Power Electronics and Design (ISLPED), pp72-75.

[8] J. Kanjanarot, K. Sitthi, and C \& Saivichit, (2006) "Energy-based Route Discovery Mechanism in Mobile Ad Hoc Networks”, ICA0T2006, pp1967-1972.

[9] Dahai Du \& Huagang Xiong, (2010) “A Location aided Energy-Efficient Routing Protocol for Ad hoc Networks", 19th Annual Wireless and Optical Communications Conference (WOCC), pp1-5.

[10] Jangsu Lee, Seunghwan Yoo \& Sungchun Kim, (2010) "Energy aware Routing in Location based Ad-hoc Networks", Proceedings of the 4th International Symposium on Communications, Control and Signal Processing (ISCCSP), pp3-5.

[11] Nen-Chung Wang \& Si-Ming Wang, (2005) "An Efficient Location-Aided Routing Protocol for Mobile Ad Hoc Networks", 11th International Conference on Parallel and Distributed Systems (ICPADS'05), Vol. 1, pp335-341.

[12] Arthi Kothandaraman, (2008) “An Energy-Efficient Transmission Power Control Protocol for Cooperative Robotics”, Master Thesis, Auburn University, Alabama, pp1-63.

[13] Yang Qin, Y.Y. Wen, H.Y. Ang \& Choon Lim Gwee, (2007) "A Routing Protocol with Energy and Traffic Balance Awareness in Wireless Ad Hoc Networks", 6th International Conference on Information, Communications \& Signal Processing, pp1-5.

[14] QualNet details :http://www.scalablenetworks.com.

[15] T.V.P.Sundararajan, K. Rajesh Kumar \& R.K. Karthikeyan, (2009) "A Novel Survey towards Various Energy Models with AD HOC on Demand Distance Vector Routing Protocol (AODV)", International Conference On Control, Automation, Communication And Energy Conservation,pp1-5.

[16] Dr. S. Karthik, S. Kannan, Dr. M.L. Valarmathi, Dr. V.P. Arunachalam \& Dr. T. Ravichandran, (2010) "An performance Analysis and Comparison of multi-hop Wireless Ad-hoc Network Routing protocols in MANET”, International Journal of Academic Research, Vol. 2, No. 4, pp119-124.

[17] Alemneh Adane, (2008) "Active Communication Energy Efficient Routing Protocol Of Mobile Ad-hoc Networks (MANETS)", A thesis submitted to the school of Graduate studies of Addis Ababa University, Ethiopia. 


\section{Authors}

Nivedita N. Joshi received the BE degree in 2008 from Pune University. She is currently a M.Tech student at College of Engineering, Pune, India which is an autonomous institute of Government of Maharashtra. She is pursuing her M.Tech in Wired and Wireless Communication. Her area of interests are wireless communication, image processing.

Radhika D Joshi received the BE degree in 1993 from Pune University and ME Degree from Pune University in 2002. She is currently a PhD student at the University of Pune. She is pursuing research in the field of Wireless Mobile Ad hoc networks energy management issues. At present she is serving as Assistant Professor at Electronics and Telecommunication Engineering department of College of Engineering Pune, which is an autonomous institute of Government of Maharashtra. Her areas of interests are wireless communication, signal processing and electronic devices and circuits. She has received grants for two research proposals from two funding agencies recently. She has published several papers in journals and international conferences. 